\title{
Editorial: Topical Volume "From Disks to Planets: The Making of Planets and Their Early Atmospheres"
}

\author{
M. Blanc ${ }^{1,2,3}$
}

Published online: 22 December 2016

(C) Springer Science+Business Media Dordrecht 2016

This topical volume entitled "From disks to planets: the making of planets and their early atmospheres" has been edited by M. Blanc, G. Herczeg, H. Lammer, V. Sterken, W. Benz, S. Udry, R. Rodrigo and M. Falanga. The papers are the result of a collaborative effort that started with an ISSI workshop organized in Beijing in August 2014 by the International Space Science Institute-Beijing (ISSI-BJ), host, and the International Space Science Institute (ISSI).

M. Blanc

Michel.blanc@issibj.ac.cn; Blanc@issibern.ch; Michel.blanc@irap.omp.eu

1 ISSI-Beijing, Beijing, China

2 ISSI, Bern, Switzerland

3 IRAP, Toulouse, France 